\title{
Breast Cancer in the Puerperium. More Questions Than Answers
}

\author{
Vouxinou A, Iatrakis $\mathbf{G}$ and Dagla $\mathbf{M}^{*}$ \\ Department of Midwifery, University of West Attica, Greece
}

*Corresponding author: Maria Dagla; Department of Midwifery, University of West Attica, Greece.

To Cite This Article: Vouxinou A, Iatrakis G, M Dagla, Breast Cancer in the Puerperium. More Questions Than Answers. 2020 - 11(1). AJBSR. MS.ID.001584. DOI: 10.34297/AJBSR.2020.11.001584.

Received: 眥 November 11, 2020; Published: 制 November 25, 2020

\begin{abstract}
Breast cancer diagnosed during puerperium and lactation is not fully understandable until today. In general, it is suggested a worse prognosis for postpartum breast cancer compared to breast cancer unrelated to pregnancy or to postmenopausal breast cancer. However, the retrospective nature of some data makes final conclusions less convincing compared to other risk factors related to breast cancer behavior. New research data showed that the screening mammography should be discussed in women aged 40 years and older.
\end{abstract}

Keywords: Breast Cancer; Puerperium; Lactation; Prognosis; Mammography

\section{Introduction}

Breast cancer related to pregnancy is considered breast cancer, which was diagnosed during pregnancy, one year after delivery or during lactation [1]. Many years ago, it was concluded that breast cancer diagnosed during puerperium and lactation tends to have worse prognosis than breast cancer in other periods of woman's life. More aggressive forms of breast cancer, negative hormone receptor status, delayed diagnosis (with increased cases with axillary nodes involvement) and delayed treatment may be responsible for these conclusions. This phenomenon is not fully understandable because hormone levels decrease markedly after expulsion of the placenta, reaching their lowest level approximately one week after birth. In nonlactating women, estrogen levels increase progressively in the second week of the postpartum period. However, in lactating women estrogen levels remain lowfor several weeks and the initial hyperprolactinemic state is associated with depressed estrogen production. In non-lactating women, 6-10 weeks following delivery, menstruation returns in most women and ovulation can be expected in the 6 th to 14 th postpartum week [2].

In general, it is suggested a worse prognosis for postpartum breast cancer compared to breast cancer unrelated to pregnancy or to postmenopausal breast cancer. However, the retrospective nature of some data makes final conclusions less convincing compared to other risk factors related to breast cancer behavior. A prospective design of such studies including and matching certain characteristics is necessary for final conclusions. As an example, age at diagnosis, tumor type (e.g. hormone receptor positive or negative), stage of the diseaseat diagnosis (e.g. axillary lymph nodes involvement or distant metastases) and the exact postpartum week of breast cancer diagnosisare some of the necessary characteristics that should be included in the comparisons of breast cancer groups. Finally, for detailed comparisons, both overall survival and diseasefree survival data should be analyzed.

In the past, it was concluded that the high concentrations of estrogen in breast fluid may explain the increased cancer risk in the puerperium. Following full-term birth and lactation, the depressed estrogen levels in breast fluid may explain the protective action of parity to breast cancer risk [3].

In the material collected for the thesis of the first author, in one patient (of a total of $>300$ patients), breast cancer was diagnosed during the postpartum period (in the 38th postpartum week). In one more patient, breast cancer was diagnosed one year and two 
months after delivery, marginally missing the definition of breast cancer related to pregnancy. In these and other cases, described in the bibliography, it is not always certain that the cancer was fully developed during the few months after delivery or, simply, it was undiagnosed during pregnancy. Finally, apart from hormonal influences, it seems that other factors (including collagen and PAPP-A) may strongly related to the etiology of postpartum breast cancer [4].

\section{Conclusion}

Considering the above data and observations, in the postpartum weeks, screening mammography should be discussed in women aged 40 years and older.

\section{conflict of interest}

There is no conflict of interest

\section{References}

1. Iatrakis G (2020) Breast cancer. In the Book: Gynaecologic Oncology Athens, Desmos Digita.

2. Berens P (2020) Overview of the postpartum period: Normal physiology and routine maternal care. UpToDate.

3. Petrakis NL, MR Wrensch, VL Ernster, R Miike, J Murai, et al. (1987) Influence of pregnancy and lactation on serum and breast fluid estrogen levels: implications for breast cancer risk. Int J Cancer 40(5): 587-591.

4. Slocum E, Germain D (2019) Collagen and PAPP-A in the Etiology of Postpartum Breast Cancer. Review Horm Cancer 10: 137-144. 